\title{
Patients, family members, and providers identified 6 components of a "good death"
}

\author{
Steinhauser KE, Clipp EC, McNeilly M, et al.In search of a good death: observations of patients, families, and providers \\ Ann Intern Med 2000 May 16;132:825-32.
QUESTION: What are the attributes of a "good death" as understood by patients, families, and providers involved in end of life care?

Design
Grounded theory.

Setting

Durham, North Carolina, USA.

\section{Participants}

75 participants (age range $26-77$ y, $64 \%$ women, $70 \%$ white, $61 \%$ Protestant) were recruited from a university medical centre, a Veterans Affairs medical centre, and a community hospice. Participants included 27 nurses, 10 social workers, 8 hospice volunteers, 6 chaplains, 6 physicians, 14 patients, and 4 bereaved family members.

Voterans Affairs Health

Services Research and

Development.

For correspondence:

Dr K E Steinhauser,

Veterans Affairs Medical Center (152), 508 Fulton Street, Durham, $N C$ 27705, USA. Fax +1 9194165838

\section{Methods}

12 focus groups of $6-8$ participants, stratified by role and race, were held over a 4 month period until data saturation occurred. Participants were asked to discuss their experiences of the deaths of family members, friends, or patients and to reflect on what made those deaths good or bad. After analysing focus group transcripts, 2 members from each group (the most and

\section{COMMENTARY}

The study by Steinhauser $e t$ al provides an important contribution to our understanding of what constitutes a "good death" from the perspective of patients, families, and providers involved in end of life care. The findings are consistent with other end of life studies, which show that appropriate biomedical care, combined with psychosocial and spiritual care, greatly promote quality of dying. ${ }^{1-3}$ The importance of being known as a "whole person" and the ability to contribute to the wellbeing of others were 2 components of a "good death" that have not been previously identified.

Although this study offers nurses direction for practice, the findings should not be arbitrarily applied to different practice settings and patient populations. The findings were primarily derived from providers and may not entirely reflect the perceptions of patients and families. Of the 75 participants, only 14 were patients and most of these were men identified from oncology and HIV clinics. Further research should address whether sex, location of care, and diagnosis influence a patient's perceptions.

The study findings provide direction for the development of nursing interventions to enhance the death experience. The findings suggest the need to continually reassure patients that pain and other symptoms will be adequately addressed. Interventions that allow patients and family members to have more involvement in healthcare decision making are important, particularly in anticipation of events that might occur in the future. Additionally, the findings suggest that healthcare providers respect the "whole person" and, if possible, facilitate the opportunity for the dying person to contribute to the wellbeing of others. Careful consideration of the 6 components identified in this study may assist nurses in ensuring a "good death" for their patients.

Kelli Stajduhar, RN, MSN Doctoral Candidate, School of Nursing University of British Columbia

Vancouver, British Columbia, Canada

1 Barnes R, Barrett C, Weintraub S, et al. Hospital response to psychosocial needs of AIDS inpatients. J Palliat Care 1993;9:22-8.

2 Davies B (editor). Fading away: the experience of transition in families with terminal illness (death, values, and meaning). Amityville, NY: Baywood Publishing, 1995.

3 Hinton J. Services given and help perceived during home care for terminal cancer. Palliat Med 1996;2:125-34. least talkative) were interviewed; no new themes emerged. Focus groups and interviews were audiotaped, transcribed, and analysed using the constant comparative method.

\section{Main findings}

Participants identified 6 components of a good death. The first, pain and symptom management, reflected fears of dying in pain and concerns about control of both current and future pain. Patient anxiety about breakthrough pain or extreme shortness of breath was alleviated with reassurance (eg, morphine drip will be provided). The second component, clear decision making, referred to the reduction of fear of pain and inadequate symptom management through communication and clear decision making. Patients felt empowered when they participated in treatment decisions. Bad deaths were often described in terms of patient feelings of being disregarded, family concerns about suffering, and provider feelings of being out of control and not able to provide good care. Often, decisions had not been discussed previously and were made in crisis situations. The third component, preparation for death, reflected the need for better preparation for the end of life. Patients wanted to know what to expect during the course of their illness and wanted to plan for events that would occur after their death (eg, planning funeral). Family members wanted to know about the physical and psychosocial changes involved in dying. Preparation of care providers was usually of a personal nature and occurred outside of formal training. The fourth component, completion, involved faith issues, life review, resolving conflicts, spending time with family and friends, and saying goodbye. Issues of faith, although highly individual, were often described as integral to healing at the end of life and became more important as physical decline occurred. Contributing to others, the fifth component, referred to the importance of allowing patients with terminal illness to contribute to the wellbeing of others, in the form of gifts, time, or knowledge. The sixth component, affirmation of the whole person, was about the importance of seeing the patient as a unique and whole person. Family members appreciated providers who did not see patients as "diseases," but understood them in the context of their lives, values, and preferences.

\section{Conclusion}

Patients, family members, and providers identified 6 common components of a good death: pain and symptom management, clear decision making, preparation for death, completion, contributing to others, and affirmation of the whole person. 